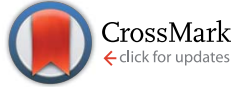

Cite this: RSC Adv., 2017, 7, 2753

Received 31st October 2016 Accepted 14th December 2016

DOI: $10.1039 / c 6 r a 26062 d$

www.rsc.org/advances

\section{Controlled release of vancomycin from 3D porous graphene-based composites for dual-purpose treatment of infected bone defects $\dagger$}

\author{
Weizong Weng, $\dot{t}^{\mathrm{a}}$ Wei Nie, $\dot{t}^{\mathrm{b}}$ Qirong Zhou, ${ }^{a}$ Xiaojun Zhou, ${ }^{\mathrm{b}}$ Liehu Cao, ${ }^{\mathrm{a}}$ Fang Ji, ${ }^{\text {a }}$ \\ Jin Cui, ${ }^{a}$ Chuanglong $\mathrm{He}^{* \mathrm{~b}}$ and Jiacan $\mathrm{Su}^{\star \mathrm{a}}$
}

Infected bone defects (IBD) are a challenging problem in orthopedic practice. Biomaterials have attracted attention as a novel approach to overcome this challenge instead of using conventional strategies. Graphene, a two-dimensional carbon allotrope, has been shown to possess multiple advantages, such as osteogenesis-promoting activity, a large surface area for drug delivery, and antibacterial activities. However, these attractive merits have not been entirely explored or introduced for the management of IBD. Herein, a novel approach using a vancomycin-laden, self-assembled, graphene-based material against IBD was developed, and the approach uses a single-stage bone graft instead of multiple procedures. Owing to the $\pi-\pi$ bonding with graphene, vancomycin can be promptly delivered in the initial stage, followed by a sustained release, which ensures the rapid elimination of an infection and provides a durable pathogen revival inhibition via the inherent antibacterial activity of graphene. Moreover, the 3D porous structure incorporated with nano-hydroxyapatite $(\mathrm{nHA})$ provided a biomimetic microenvironment favored by cell adhesion and osteogenic differentiation. Further evaluation of this system in an IBD animal model demonstrated prompt control of infection and promotion of bone regeneration. These results revealed the potential of this approach to be an effectual solution for IBD treatment and a new area of graphene application in the future.

\section{Introduction}

Infected bone defect (IBD) refers to the loss of bone segments combined with infection, ${ }^{\mathbf{1 , 2}}$ and IBD usually results from severe open fractures due to traffic accidents and combat injuries. ${ }^{3}$ The causative injuries are often associated with severely damaged soft tissue and local blood supply, contaminated wounds, and a high occurrence of large volumetric bone loss. ${ }^{4}$ Conventional systematic administration of antibiotics yields a limited success rate, which is mainly attributed to the dramatically damaged local blood supply from the causative injuries and the formation of a bacteria biofilm that can impair the antibiotic efficacy. ${ }^{4,5}$ Furthermore, bacteria propagation and damaged vascularity contribute to the integrated effect that is responsible for restraining the process of bone regeneration..$^{6,7}$

\footnotetext{
${ }^{a}$ Department of Orthopaedics Trauma, Changhai Hospital, Second Military Medical University, No. 168 Changhai Road, Shanghai 200433, China. E-mail: chjifang1962@126.com; drsujiacan@163.com

${ }^{b}$ College of Chemistry, Chemical Engineering and Biotechnology, State Key Laboratory for Modification of Chemical Fibers and Polymer Materials, Donghua University, 2999 North Renmin Road, Shanghai 201620, China.E-mail: hcl@dhu.edu.cn

$\dagger$ Electronic supplementary information (ESI) available. See DOI: 10.1039/c6ra26062d

\$ These authors contributed equally to this work.
}

Because of the grievous morbidity suffered by the population afflicted with IBD and the subsequent disabilities, endeavors have been made to develop novel biomaterial systems to eradicate infection and simultaneously facilitate bone regeneration. However, devising such an on-demand biomaterial system is complicated because it requires taking multiple aspects into account, such as biocompatibility, promotion of bone regeneration, controlled release of antibiotics and inherent antimicrobial ability for prolonged pathogen inhibition. ${ }^{8}$

Local controlled release kinetics play a vital role in devising biomaterials for improved bone infection treatment. ${ }^{9-11}$ The kinetics allow the production of the effective local antibiotic concentration, which is difficult to achieve with a systemic antibiotic therapy owing to the damaged vascularity and necrotic bone tissue, reduce the systemic toxicity, ${ }^{\mathbf{1 2 - 1 4}}$ and contribute to the prolonged defence against bacteria propagation, which facilitates new bone regeneration unhindered by infection. ${ }^{7,15}$

Thus far, various local delivery systems developed from biomaterials, such as poly(D,L-lactide-co-glycolide acid) (PLGA), poly(L-lactic acid) (PLLA) and silica sol gels, have achieved effectual drug loading and sustained release kinetics for antibiotics in the time range of weeks. ${ }^{16-19}$ However, their osteoconductivity deficiency remains a limit for further application. Osteoconductive biomaterials like hydroxyapatite (HA), silica- 
calcium phosphate composites and bioactive glass were constructed in drug delivery systems, and while accelerated bone formation besides sustained drug release was achieved, ${ }^{\mathbf{2 0 - 2 3}}$ the lack of inherent antibacterial activity rendered the graft site vulnerable to bacterial adhesion and propagation after exhaustion of the antimicrobials. ${ }^{24}$

Considering the disadvantages of the biomaterials in current applications and the specific demands for infected bone defect treatment, it is critical to choose an alternative biomaterial that has integrated abilities comprising osteogenesis, local drug delivery and inherent antibacterial activity.

Graphene materials are attractive candidates as twodimensional, atomically thin carbon allotropes. First, the ability of graphene to promote osteogenesis has been demonstrated along with its family of nanomaterials, i.e., graphene oxide (GO) and reduced graphene oxide (RGO). ${ }^{25,26}$ Composite scaffolds functionalized by graphene materials were fabricated via various methods along with hydroxyapatite (HA), gelatin and PLGA as potential biomimetic bone substitutes. ${ }^{27-30}$

Moreover, graphene has been widely used in drug delivery systems owing to its large specific surface area and $\pi$-electronrich structure. ${ }^{31-33}$ For example, GO was used for the controlled release of doxorubicin (DOX) in localized, anti-cancer therapy because of the strong bond between DOX and GO from a $\pi-\pi$ stacking interaction, ${ }^{33}$ which is a non-covalent bond that facilitates the sustained release of drugs. ${ }^{34}$ Similar to DOX in its basic aromatic moiety, vancomycin (VA) is a glycopeptide antibiotic usually utilized against Gram-positive bacteria, specifically $S$. aureus and its methicillin-resistant counterparts ${ }^{35}$ that are generally encountered in IBD. ${ }^{36-38}$ Considering its evident ototoxicity and nephrotoxicity via intravenous administration, ${ }^{39,40}$ localized and controlled deployment of this antibiotic is critical for more secure effects at therapeutic concentrations. To date, the exploration of graphene material has been limited to use as a nanocarrier of vancomycin because of its high absorption ability for vancomycin, ${ }^{\mathbf{4 1}}$ and the potential of graphene for infected bone defect management is unknown.

Ultimately, an inherent antibacterial activity was also demonstrated by graphene, and the mechanism was cell membrane damage of the bacteria via the sharp edges of the nanowalls and oxidation stress. ${ }^{\mathbf{4 2 , 4 3}}$ Combining the inherent antibacterial activity and advantages in drug loading, graphene in composites can deliver enhanced and extended antibacterial activity, and accelerated bone repair is predicted with osteoconductivity additives..$^{30,44-47}$

Herein, we developed a novel approach against infected bone defect using graphene-based composites, and the schematic is depicted in Fig. 1. Briefly, vancomycin was incorporated into a $3 \mathrm{D}$ porous composite that was self-assembled by RGO and osteoconductive nano-hydroxylapatite (nHA), providing localized deployment of the antibiotic against the invading pathogens in a sustained manner to avoid bolus release and simultaneously creating a favored biomimetic microenvironment for cell adhesion and osseous regeneration.

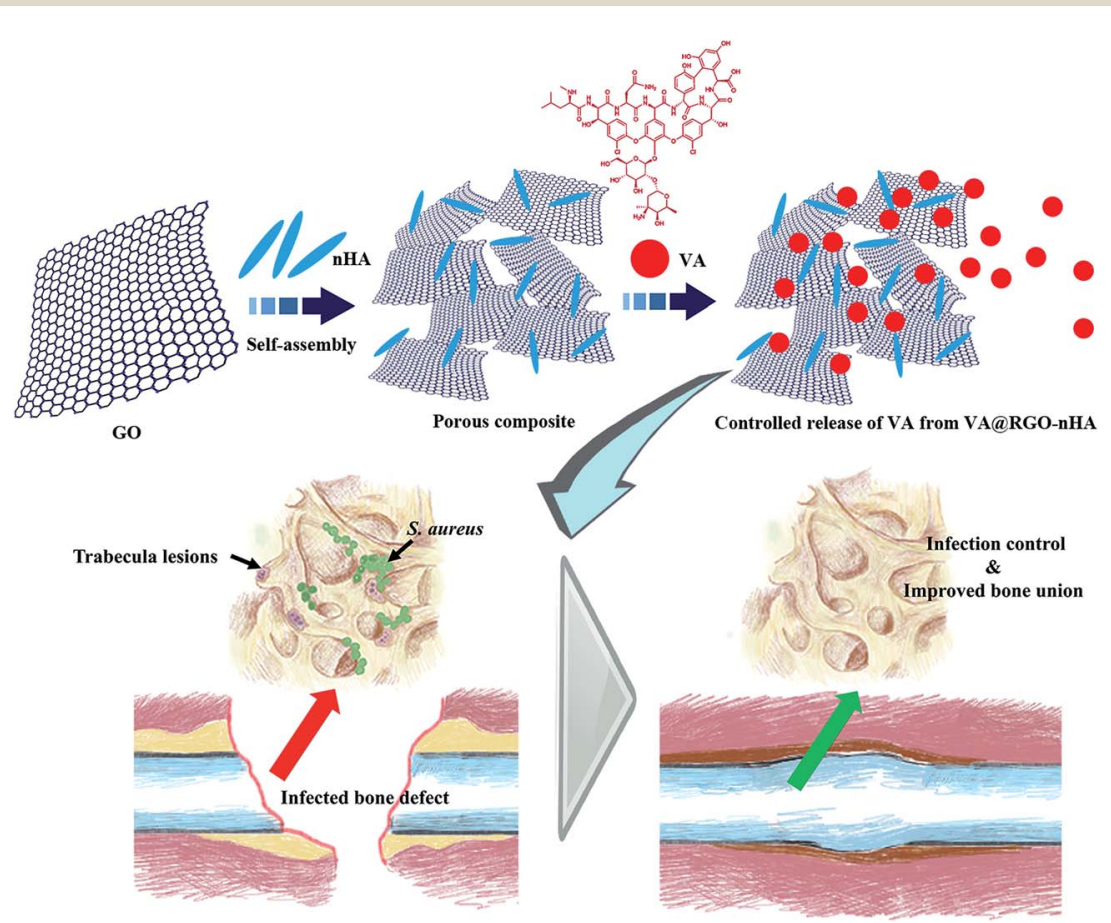

Fig. 1 Schematic of the fabrication of the reduced graphene oxide/nano-hydroxyapatite (RGO-nHA) 3D porous composite by a self-assembly method and loading of the VA onto the scaffold. VA can be released via a controlled profile from the porous scaffold, which allows for durable antibacterial activity along with the inherent antimicrobial ability of graphene. While the incorporated formulation of the composite provides an osteoconductive and a porous biomimetic microenvironment favored by cell adhesion and osteogenic differentiation, thus facilitating an accelerated bone regeneration process unhindered by infection. Abbreviations: GO, graphene oxide; nHA, nano-hydroxyapatite; RGO, reduced graphene oxide; VA, Vancomycin; S. aureus, Staphylococcus aureus. 
The micromorphology and physicochemical properties of the VA-laden RGO-nHA composite scaffold (VA@RGO-nHA) were characterized via scanning electron microscopy (SEM) and Fourier transform infrared spectroscopy (FTIR). The release profile for VA from the composite was assessed, and the in vitro antibacterial capacity against $S$. aureus was evaluated. Cell viability, morphology and osteogenic differentiation were appraised by seeding rat bone mesenchymal stem cells (rBMSCs) onto the composite scaffolds. Moreover, a rabbit radius bone defect model with an infection induced by $S$. aureus was established to determine the capacity of the RGO-nHA composite in IBD treatment, while providing a controlled release of antibiotics. We demonstrated that this constructed approach achieved prompt infection control, and accelerated bone repair due to eradication and sustained inhibition of the infectious pathogens as well as a rational device of the scaffold both in osteoconductive formulation and $3 \mathrm{D}$ porous structure.

\section{Materials and methods}

\subsection{Materials}

$\mathrm{H}_{2} \mathrm{SO}_{4}(98 \%), \mathrm{KMnO}_{4}, \mathrm{H}_{2} \mathrm{O}_{2}(30 \%)$, tert-butanol, and polymethyl methacrylate (PMMA) were purchased from Sigma-Aldrich Trading Co., Ltd. (Shanghai, China). Expandable graphite flakes were ordered from ChengYang Graphite Products Co., Ltd. (Qingdao, China). Vancomycin hydrochloride, dexamethasone, $\beta$-glycerophosphate, ascorbic acid, paraformaldehyde, and nHA were received from Aladdin Chemistry Co., Ltd. (Shanghai, China). Dulbecco's Modified Eagle's Medium/ nutrient mixture F-12 (DMEM F-12), foetal bovine serum (FBS), penicillin, streptomycin, and phosphate buffer saline (PBS) were purchased from Gibco (Grand Island, USA). ALP and BCA Protein assay kits were received from the Beyotime Institute of Biotechnology (Shanghai, China). Calcein AM was obtained from Yeasen Biotech Co., Ltd. (Shanghai, China). New Zealand white rabbits were ordered from Slac Laboratory Animal Co., Ltd. (Silaike Inc., Shanghai, China). The water used in all experiments was purified using a Milli-Q water purification system (Millipore, Bedford, MA) with a resistivity of 18.2 $\mathrm{M} \Omega \mathrm{cm}$. All other chemicals were of analytical grade and were obtained from Sinopharm Chemical Reagent Co., Ltd. (Shanghai, China).

\subsection{Synthesis of VA@RGO-nHA}

The GO sheets were prepared from expandable graphite flakes according to a previously reported method. ${ }^{46}$ The RGO-nHA porous scaffolds with a $20 \%$ nHA loading ratio were synthesized via self-assembly of GO and nHA, as previously described. ${ }^{30}$ Briefly, GO was suspended and ultrasonicated in $80 \mathrm{ml}$ of ultrapure water to obtain a $3.5 \mathrm{mg} \mathrm{ml}^{-1}$ transparent colloidal solution. Four millilitres of a nHA suspension with a concentration of $7 \mathrm{mg} \mathrm{ml}^{-1}$ was added into a GO suspension to obtain the nHA loading mixture, which was then ultrasonicated in an ice bath. The self-assembly process was induced by heating the suspension to $200{ }^{\circ} \mathrm{C}$ in a stainless steel reaction kettle for $3 \mathrm{~h}$. Finally, the reaction products were freeze-dried to obtain the
20\% nHA incorporated RGO (RGO-nHA) 3D porous scaffold. VA was used for drug loading onto the RGO-nHA. The loading process was achieved by mixing different concentrations of VA with an aqueous solution of RGO-nHA $\left(1 \mathrm{mg} \mathrm{ml}^{-1}\right)$ and then lyophilizing the solutions to obtain 1\%,2\%, and 4\% VA@RGOnHA for further experiments.

\subsection{Characterization}

Attenuated total reflectance-Fourier transform infrared spectroscopy (ATR-FTIR, Nicolet Nexus 670 FTIR spectrometer, Thermo Electron Co., USA) was utilized for Fourier transform infrared analysis of the functional groups of the scaffolds, covering an infrared range of $500-4500 \mathrm{~cm}^{-1}$. The micromorphology of the VA@RGO-nHA porous scaffolds was characterized using a scanning electron microscope (SEM, JSM$6701 \mathrm{~F}$, JEOL, Japan) with an acceleration voltage at $10 \mathrm{kV}$. Briefly, the prepared samples were placed on the stage and a thin layer of Pt was sputter-coated onto the surface to prevent abnormal charging.

\subsection{In vitro VA release from VA@RGO-nHA}

The drug release profiles of the VA@RGO-nHA scaffolds with different VA concentrations were analysed by recording the absorbance of VA at $214 \mathrm{~nm}$ with a Lambda $25 \mathrm{UV}$-Vis spectrophotometer (Perkin Elmer, USA). Briefly, $50 \mathrm{mg}$ of the VA@RGO-nHA scaffolds were placed in a dialysis bag and immersed in a tube containing $10 \mathrm{ml}$ of PBS at a pH of 7.4. This was shaken for different time periods using a water bath constant temperature vibrator at $37^{\circ} \mathrm{C}$ with a vibrating speed of $180 \mathrm{rpm}$. At pre-designated time points, $200 \mu \mathrm{l}$ of the release medium samples were retrieved and replenished with an equal volume of PBS to maintain a constant volume. The experiment was repeated in triplicate.

\subsection{Mesenchymal stem cell isolation and culture}

Rat bone mesenchymal stem cells (rBMSCs) from 1-week-old female rats were harvested and cultured following the previously reported method. ${ }^{48}$ Specifically, the rats were sacrificed with an overdose of isoflurane, and the femoral cavities were cut open. Foetal bovine serum (10\%) (FBS, Gibico, Thermofisher scientific, USA) in phosphate-buffered saline (PBS, Hyclone, China) was utilized to rinse the marrow cavity; thus, the cells within the marrow cavity were flushed out and collected. After centrifugation for $10 \mathrm{~min}$ at $1000 \mathrm{rpm}$, the cell suspension was re-suspended in DMEM/F12 (Hyclone, China) containing 15\% FBS, $100 \mathrm{U} \mathrm{ml}^{-1}$ penicillin (Gibco, Grand Island, USA), and $100 \mathrm{mg} \mathrm{ml}^{-1}$ streptomycin (Gibco, Grand Island, USA). Subsequently, a cell culture flask was applied for cell seeding in a humidified atmosphere with $5 \% \mathrm{CO}_{2}$ at $37{ }^{\circ} \mathrm{C}$. When the rBMSCs were passed three times, the cells were used in the following experiments.

\subsection{Cell proliferation and cytocompatibility}

The cell proliferation on VA@RGO-nHA scaffolds was examined with a cell number counter (Countstar, RuiYu Biotech, 
Shanghai, China); calcein AM (Yeasen Biotech, Shanghai, China) fluorescence staining was used for identification of live cells. Green fluorescence indicated live cells. Scaffolds underwent ultraviolet radiation sterilization and were placed into a 48-well culture plate. For cell proliferation evaluation, cells with a density of $2 \times 10^{4}$ cells per well were seeded in the well. The cells seeded on the scaffolds were retrieved at 2, 4 and 8 days, washed using PBS 3 times, and added to $0.2 \mathrm{ml}$ of Tripsin. The cell proliferation was then determined using the cell number counter. For fluorescence staining, each well was supplemented with a growth medium $(300 \mu \mathrm{l})$ and calcein AM (500 $\mathrm{nM}$ ). After incubation for 30 minutes, the live cells on the scaffolds were observed with a fluorescence microscope (Olympus Bx61, Japan) after 4 and 8 days' incubation period.

\subsection{Morphology of rBMSCs on scaffolds}

SEM (SEM, Hitachi TM-1000, Japan) and CLSM (Carl Zeiss LSM 700, Germany) were used for observation of the morphology of rBMSCs cultured on scaffolds with different VA concentrations with RGO-nHA as a control. rBMSCs with a density of $5 \times 10^{4}$ cells per well were seeded and co-cultured with the scaffolds in a 24-well plate overnight at $37{ }^{\circ} \mathrm{C}$ with a $5 \% \mathrm{CO}_{2}$ atmosphere, and the medium was replaced with an osteogenic medium. At pre-determined time intervals ( 4 day, 8 day), cells were rinsed 3 times with PBS, fixed with $2.5 \%$ glutaraldehyde, dehydrated in gradient ethanol solutions $(30 \%, 50 \%, 70 \%, 80 \%, 90 \%, 95 \%$, and $100 \%)$, soaked in gradient tertiary butanol solutions $(25 \%$, $50 \%$, and $100 \%$ ), lyophilized in a vacuum freeze-drier, and finally observed via SEM. For CLSM observation of cell spreading, the cells grown on the scaffolds after a predetermined time interval were first washed with PBS three times and then fixed with $3.7 \%$ formaldehyde for 10 minutes at room temperature. After being rinsed three times with PBS, the cells were permeabilized with $0.1 \%$ Triton X-100 in PBS for $10 \mathrm{~min}$ and washed using PBS again three times. Afterwards, rhodamine-phalloidin (Molecular Probe, ThermoFisher Scientific, USA) and 40, 60-diamidino-2-phenylindole (DAPI, Molecular Probe, ThermoFisher Scientific, USA) staining was carried out for visualization of the actin cytoskeleton and nuclei, respectively.

\subsection{Alkaline phosphatase (ALP) activity}

For osteogenic differentiation, the osteogenic medium was prepared as previously reported. ${ }^{49}$ A total of $1 \times 10^{4}$ rBMSCs were seeded onto the scaffolds and cultured in the growth medium. The medium was then replaced with the osteogenic medium at $80 \%$ confluence, and the osteogenic medium was changed every two days. For the ALP activity evaluation, the suspension of rBMSCs was seeded in a 96-well plate and cocultured with a colloidal dispersion of RGO $\left(10 \mu \mathrm{g} \mathrm{ml}{ }^{-1}\right)$, RGO-nHA (10 $\left.\mu \mathrm{g} \mathrm{ml}^{-1}\right)$, 1\% VA@RGO-nHA $\left(10 \mu \mathrm{g} \mathrm{ml}^{-1}\right)$ and 2\% VA@RGO-nHA scaffolds $\left(10 \mu \mathrm{g} \mathrm{ml}{ }^{-1}\right)$ at $37{ }^{\circ} \mathrm{C}$ under $5 \% \mathrm{CO}_{2}$. At pre-determined time intervals ( 0,7 , and 14 days), the cells were rinsed twice with PBS; $300 \mu \mathrm{L}$ of cell lysis buffer was then added to the well, and the scaffolds were homogenized at $4{ }^{\circ} \mathrm{C}$. The mixture was subjected to sonication and was centrifuged at
$8000 \mathrm{rpm}$ for $10 \mathrm{~min}$. ALP activity and total protein from the supernatant were determined using the ALP and BCA assay kits. The relative ALP activity was normalized to the protein concentration of each sample.

\subsection{In vitro antibacterial activity against $S$. aureus}

As $S$. aureus is a Gram-positive bacterium and the main pathogenic bacteria that causes infected bone defect and osteomyelitis, ${ }^{50}$ S. aureus (ATCC 25923, USA) was chosen as the target bacteria to evaluate the antibacterial activity of VA@RGO-nHA both in a liquid and solid medium. For the following experiments, frozen $S$. aureus was activated with a fresh TSB medium with gentle shaking in a $37{ }^{\circ} \mathrm{C}$ humidified atmosphere. The tested scaffold samples were sterilized with ultraviolet radiation for $3 \mathrm{~h}$ before use.

2.9.1 Antibacterial analysis in liquid medium. S. aureus was first added to $100 \mathrm{ml}$ of liquid TSB medium with a bacterial density of $2 \times 10^{5}$ per ml for each test tube. VA@RGO-nHA with VA concentrations of $1 \%, 2 \%$, and $4 \%$ were then added to each test tube. The bacteria-scaffold mixtures were co-incubated at $37{ }^{\circ} \mathrm{C}$ with gentle shaking $(180 \mathrm{rpm})$. At pre-determined time intervals, the co-incubated mixtures were analysed with a cell counter to quantify the antibacterial efficiency. The experiment was repeated in triplicate.

2.9.2 Antibacterial analysis on a solid medium. The inhibition zone of $S$. aureus cultured on a TSB agar plate was assessed via a Kirby-Bauer assay. ${ }^{51}$ Briefly, the $S$. aureus suspension (100 ml, $10^{8} \mathrm{CFU}$ per $\mathrm{ml}$ ) was first spread onto the agar plates, and the porous scaffolds (RGO-nHA, 1\% VA@RGOnHA, 2\% VA@RGO-nHA, 4\% VA@RGO-nHA) with a mass of $4 \mathrm{mg}$ were pasted onto the agar plate. The bacteria were then incubated at $37^{\circ} \mathrm{C}$ for $24 \mathrm{~h}$, and the inhibition zone for each sample on the plate was photographed and quantitatively analysed using the Image image software (National Institutes of Health, USA) for the measurement of the inhibition zones.

\subsection{In vivo evaluation}

The capacity of the VA@RGO-nHA scaffold in repairing infected bone defects was examined further. The in vivo study was conducted according to the NIH guidelines for the care and use of laboratory animals (NIH Publication No. 85-23 Rev. 1985) and was assessed by the ethics committee in animal experimentation of the Research Center for Laboratory Animal of the Second Military Medical University, Shanghai, China.

2.10.1 Establishment of the infected bone defect model. Fifty-four male New-Zealand rabbits with an average weight of 3 $\mathrm{kg}$ were purchased from Slac Laboratory Animal Corp. (Shanghai, China). Before model establishment, the initial data, such as WBC and CRP, were collected through the rabbits' marginal ear veins with ethylenediaminetetraacetic acid (EDTA) tubes. The rabbits were intraperitoneally anesthetized with $3 \%$ pentobarbital sodium $\left(30 \mathrm{mg} \mathrm{kg}^{-1}\right)$. The right forearms in the operation zone were shaved and sterilized with a povidoneiodine solution and $70 \%$ ethanol, and a longitudinal incision of approximately $15 \mathrm{~mm}$ in length was made at the anteromedial aspect. The vessels and muscle tendons were 
cautiously blunt-dissected to further visualize the radius bone. An oscillating saw (Stryker Cooperation, Kalamazoo, Michigan, USA) was used to remove a $10 \mathrm{~mm}$ segment from the middle of the radius shaft. After irrigation with a saline solution in the bone void, $0.5 \mathrm{ml}$ of a saline suspension with an $S$. aureus (ATCC 25923 , USA) concentration of $2.5 \times 10^{6} \mathrm{CFU}$ per $\mathrm{ml}$ was injected into the defect site and the bone marrow cavity. Sodium morrhuate $(0.2 \mathrm{ml})$ (Xinyi Jinszhu Pharmacy Co. Ltd, Shanghai, China) was then added, and sterilized medical bone wax (B. Braun Surgical, Aesculap Ag \& Co., Germany) was utilized to seal the cavity. The incision was closed with 1 \# stitches (Ethicon, USA). The WBC and CRP levels of the rabbits that underwent the modelling surgery were recorded. The rabbits were then randomly assigned into three groups: A, the RGO group; $\mathrm{B}$, the RGO-nHA group; and C, the VA@RGO-nHA group.

Verification of the rabbit infected bone defect model was conducted based on the WBC and CRP variations, wound observation, X-ray photograph and histological changes. Rabbits in each group were randomly euthanized 2 weeks after the modelling surgery, and bone tissue slices were retrieved, fixed in $4 \%$ paraformaldehyde, dehydrated in a gradient series of ethanol solutions $(70 \%, 75 \%, 80 \%, 85 \%, 90 \%, 95 \%, 100 \%)$ for $24 \mathrm{~h}$, and embedded in polymethylmethacrylate. Serial sagittal sections with a thickness of $100 \mu \mathrm{m}$ were obtained using a microtome (EXAKT310, Germany) and then polished to a thickness of approximately $40 \mu \mathrm{m}$. The sections of undecalcified specimens were then subjected to haematoxylin and eosin (H\&E) staining for observation. Other harvest bone splices were soaked in PBS, and the resulting suspension was added to the agar plate and cultured overnight for observation of the bacteria colony formation.

2.10.2 Scaffold implantation and subsequent evaluations. Scaffold implantation was conducted 2 weeks after the modelling surgery. After anaesthesia, lavage and debridement, a similar approach from the same incision, was applied. The scaffolds were sterilized prior to use. The rabbits of each group were implanted with the same amount of the sterilized scaffolds (RGO, RGO-nHA, and VA@RGO-nHA, respectively) in the defect site. Afterwards, the incision was sutured and covered with a sterilized bandage. Changes in weight, WBC count, and CRP level were recorded weekly. Bone regeneration was evaluated by micro-computed tomography (SkyScan1076, Bruker micro-CT, Germany). Three-dimensional CT images were reconstructed with SkyScan CTVOX 2.1 software (Bruker micro$\mathrm{CT}$, Germany), and the bone volume (BV) and bone mineral density (BMD) were analysed accordingly. Further insight into the microstructure of the regenerated bone was achieved by histological analysis similar to the methods described above.

\subsection{Statistical analysis}

All of the quantitative data were expressed as the means \pm standard deviation (SD). Statistical analyses and graphical output production were carried out using a one-way analysis of variance (one-way ANOVA) with the assistance of Origin 9.0 (OriginLab Software, USA). A value of $p<0.05$ was considered statistically significant.

\section{Results and discussion}

\subsection{Synthesis and characterization of VA@RGO-nHA composites}

The nHA particles were first mixed with the GO suspension (lateral dimensions $0.5-5 \mu \mathrm{m}$ ), resulting in homogeneous hydrocolloids with a $20 \%$ mass ratio of nHA to GO. The selfassembly process was triggered by heating the mixture to $200{ }^{\circ} \mathrm{C}$ for $3 \mathrm{~h}$, and the reduction of GO flakes was achieved simultaneously. A free-standing hydrogel similar to that obtained in previous reports was acquired..$^{\mathbf{3 0}, 46}$ Afterwards, a $10 \mathrm{ml}$ solution with graded concentrations of VA was added to an aqueous solution of RGO-nHA at $4{ }^{\circ} \mathrm{C}$ and placed in an ice bath overnight. Finally, the hydrogel was immersed in liquid nitrogen followed by freeze-drying for $24 \mathrm{~h}$ to obtain a porous scaffold.

The morphology and physicochemical properties of RGOnHA were further analysed. As shown in the SEM microphotographs (Fig. 2), the prepared scaffold exhibited a porous structure with diameters ranging from 1-12 $\mu \mathrm{m}$ (Fig. 2i-1), which were within the range of the mesoporous pores. ${ }^{52}$ Furthermore, the scaffold with the VA loading was not observed with evident variations in the micro-morphology, and there was no VA crystallization observed in the scaffolds loaded with different concentrations of VA $(1 \%, 2 \%$, and $4 \%)$, which indicated that VA was uniformly absorbed onto the interfaces of the RGOnHA. As a result, the incorporation of nHA had an essential impact on the scaffold and facilitated the interconnected microporous structure formation; loading VA onto the scaffold did not compromise the fine construction.

The successful loading of VA onto the RGO-nHA surface and the synthesis of the composite were also confirmed qualitatively via FTIR spectroscopy (Fig. 3). The bonds that emerged in the GO possessed vibrations at $1720(\mathrm{C}=\mathrm{O}) \mathrm{cm}^{-1}$. The peaks for the oxygen functional groups disappeared in the RGO, which indicated the reduction of the GO. Moreover, the characteristic absorptions for nHA were 958 and $1080 \mathrm{~cm}^{-1}$ for $\mathrm{P}-\mathrm{O}$. The absorption bands for VA were at $1655 \mathrm{~cm}^{-1}(\mathrm{C}=\mathrm{O}), 1504 \mathrm{~cm}^{-1}$ $(\mathrm{C}=\mathrm{C})$, and $1231 \mathrm{~cm}^{-1}$ (phenols). In the VA-loaded RGO-nHA scaffolds (VA@RHA), the composite presented the characteristic peaks of VA (amide I, $1655 \mathrm{~cm}^{-1}$ ), $\mathrm{nHA}\left(\mathrm{P}-\mathrm{O}, 1080 \mathrm{~cm}^{-1}\right.$ ), and RGO $\left(1400 \mathrm{~cm}^{-1} \mathrm{C}-\mathrm{H}\right)$. The FTIR data qualitatively verified the loading of VA onto the RGO-nHA.

\subsection{Release profile of VA from the composite scaffold}

The release profiles of VA from the composite scaffolds are shown in Fig. 4 . It is clear that the VA@RGO-nHA scaffolds (1\%, $2 \%$, and $4 \% \mathrm{VA}$ ) exhibited an evident initial burst release within the first $6 \mathrm{~h}$. Specifically, the initial burst phenomenon was the most prominent in the 4\% VA@RGO-nHA, followed by the $2 \%$ VA@RGO-nHA and 1\% VA@RGO-nHA, with cumulative VA releases of approximately $82 \%, 70 \%$, and $59 \%$, respectively. The initial burst release was followed by a successive sustained release, which was more significant in 1\% VA@RGO-nHA and 2\% VA@RGO-nHA. This can be explained by the fact that despite the hydrophilic nature of VA and the strong bonding 
RGO-nHA

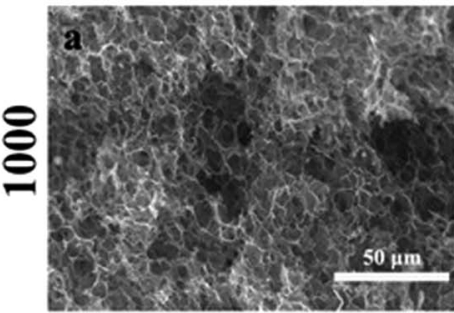

1\%VA@RGO-nHA 2\%VA@RGO-nHA

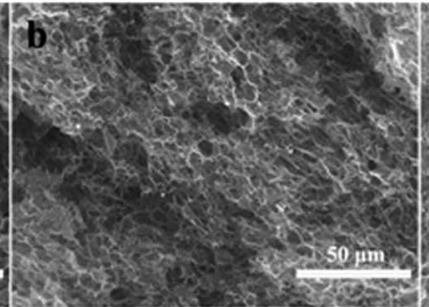

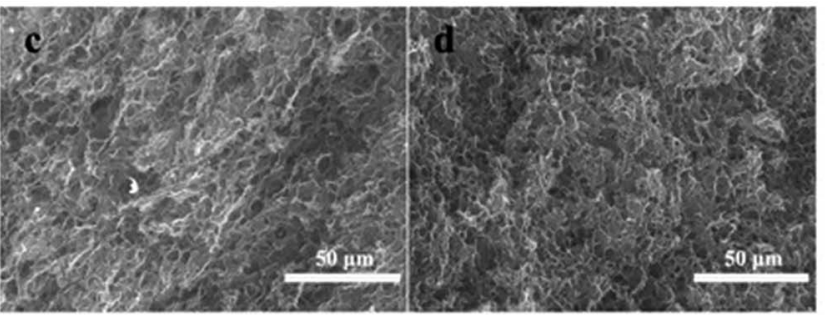
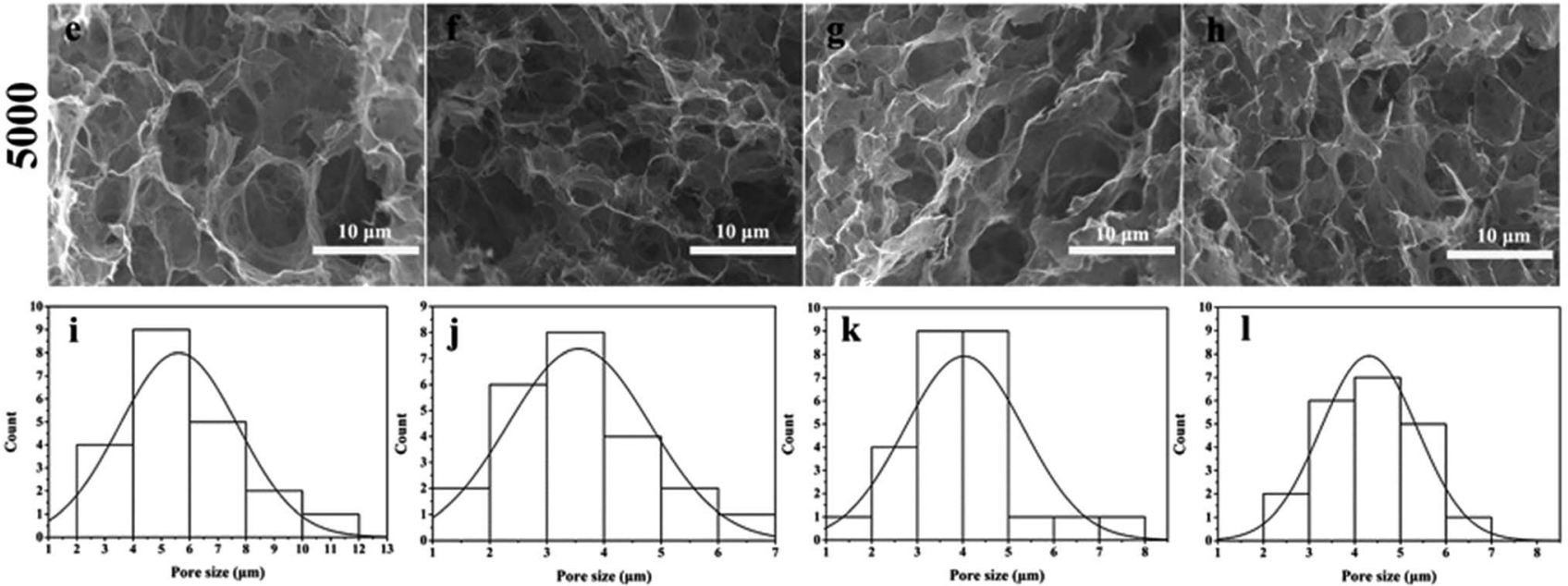

Fig. 2 SEM photographs and pore size distributions of the composite scaffolds. (a-d) Scaffold morphology under $1000 \times$ magnification, scale bar $=50 \mu \mathrm{m}$. (e-h) Scaffold morphology under 5000 $\times$ magnification, scale bar $=10 \mu \mathrm{m}$. (i-l) Pore diameter distributions of the scaffolds.

capacity of the RGO with the hydrophilic groups via $\pi-\pi$ stacking and van der Waals interactions, ${ }^{33}$ the marginal free VA molecules around the RGO sheets lack a specific binding affinity, and the van der Waals interactions were relatively low. The remaining VA molecules were adsorbed more tightly on the surface of the RGO, thus contributing to the subsequent sustained release phenomenon. These results indicated a favorable release profile for VA from the constructed composite since a fast antibiotic initial release, combined with a sustained release, is required for bone infection treatment. ${ }^{53}$

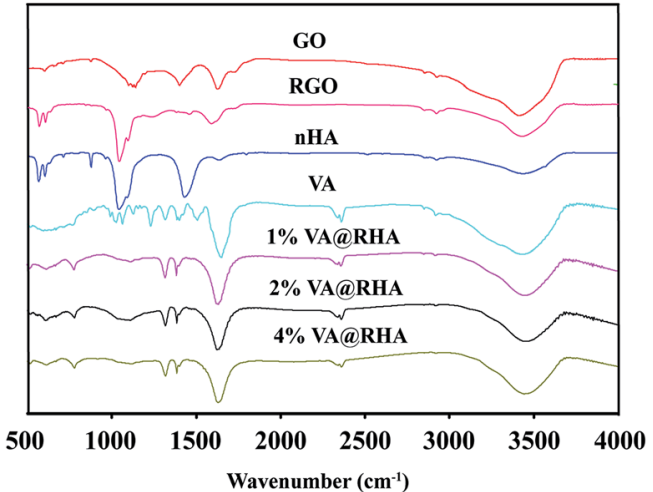

Fig. 3 FTIR spectra of GO, RGO, nHA, free VA, and VA@RGO-nHA (1\%, $2 \%$ and $4 \% \mathrm{VA}$ ).

\subsection{Cell viability and osteogenic differentiation}

The effects of RGO-nHA with different VA concentrations on the proliferation of rBMSCs were evaluated with RGO-nHA as the control (Fig. 5). As the incubation proceeded, a significant increase in the cell proliferation on scaffolds was observed. In particular, although the cells on the scaffolds loaded with $1 \%$

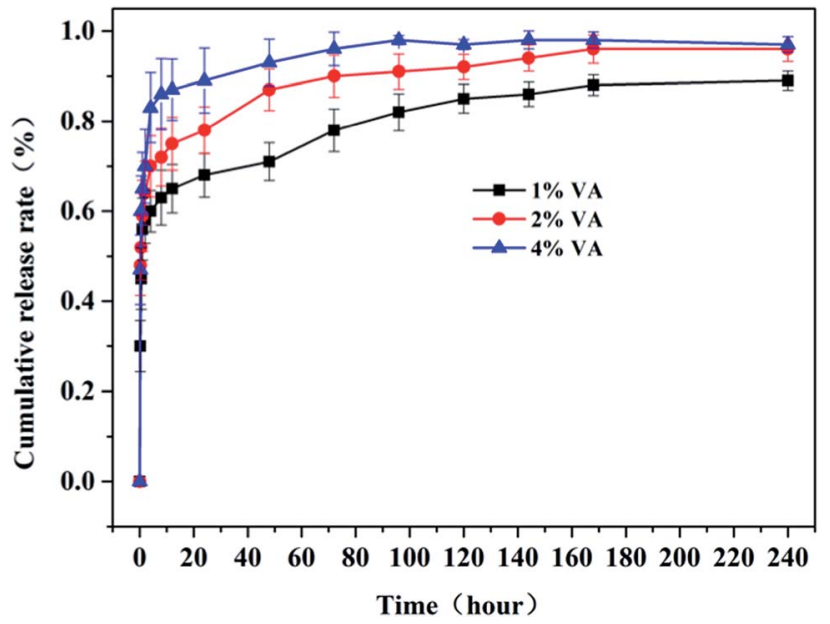

Fig. 4 In vitro vancomycin release profile of the $1 \% \mathrm{VACRGO}-\mathrm{nHA}$ (blue line at the top), 2\% VA@RGO-nHA (red line at the centre) and 4\% VA@RGO-nHA (black line at the bottom) scaffolds incubated in PBS with a $\mathrm{pH}$ of 7.3 at $37^{\circ} \mathrm{C}$. 


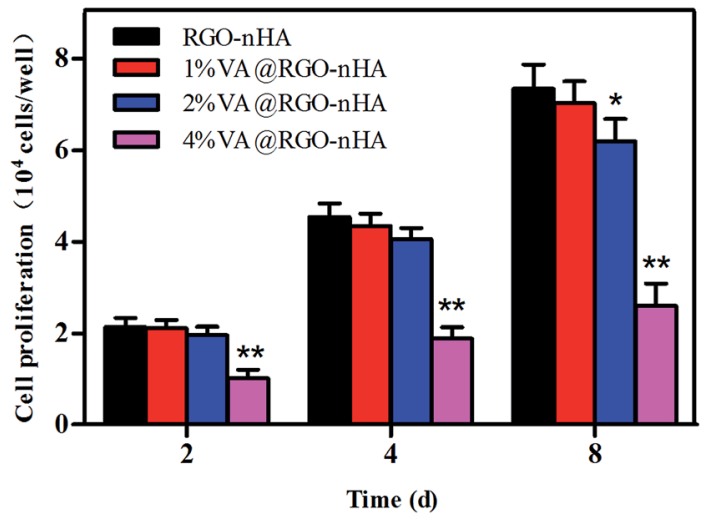

Fig. 5 Proliferation of rBMSCs cultured on RGO-nHA and $1 \%, 2 \%$, and $4 \%$ VA@RGO-nHA. Statistical significance is indicated and compared to the RGO-nHA group $(n=5, * p<0.05 ; * *<0.01)$

and $2 \%$ VA presented slightly lower proliferation rates compared to those of the RGO-nHA, this difference did not achieve a statistical significance on days 2 or $4(p>0.05)$, indicating the non-cytotoxicity of the scaffold. On day 8 , a significant disparity in the cell proliferation between 2\% VA@RGOnHA and RGO-nHA was detected, and the cells on the $4 \%$ VA@RGO-nHA were clearly suppressed. $(p<0.01)$.

The density of the rBMSCs co-cultured with the scaffolds was further examined using live cell staining. Fig. 6 shows the fluorescence photographs of cells grown on scaffolds after 4 and 8 days. Similar to the cell proliferation results, a dense distribution of cells was observed in the scaffolds loaded with $1 \%$ and $2 \%$ VA, suggesting their excellent cytocompatibility and facilitated cell attachment and growth owing to the interconnected porous structure, whereas the cell cultures on the $4 \%$ VA@RGO-nHA appeared to be distributed sporadically due to cytotoxicity at a high concentration of VA.

Fluorescence photographs of rBMSCs after 4 days of culture on the scaffolds are presented in Fig. 7. F-actin and the nuclei are indicated with red and blue, respectively. In comparison, the cells appeared to be distributed more sporadically on $4 \%$ VA@RGO-nHA than on RGO, 1\% VA@RGO-nHA, or 2\% VA@RGO-nHA (Fig. 7a, c, e and g), which was in accordance with the proliferation results. In the higher magnification images (Fig. 7b, d, f and h), better cell spreading was also revealed with more filopodia extensions and intercellular connections on RGO-nHA, 1\% VA@RGO-nHA, and 2\% VA@RGO-nHA compared to 4\% VA@RGO-nHA. Fig. 8 shows a more detailed cell morphology photographed using SEM. Better cell spreading and attachment were also observed on the lower VA-loaded scaffolds. After a 4 day culture period, the rBMSCs exhibited a clearly elongated morphology on RGOnHA, 1\% VA@RGO-nHA, and 2\% VA@RGO-nHA, but they presented round shapes on 4\% VA@RGO-nHA. More satisfactory cell spreading and attachment were observed on RGO-nHA and 1\% VA@RGO-nHA, as the cells presented more filopodia extensions. After an 8 day culture, the cells exhibited a dense distribution over RGO-nHA and 1\% VA@RGO-nHA with flat and broad shapes and larger areas, and the filopodia extensions appeared confluent, which indicated that this concentration of VA did not compromise cell attachment. In sharp contrast, the rBMSCs cultured on 4\% VA@RGO-nHA presented a less dense distribution and spread the least, proving the deleterious effect of high concentrations of VA on cellular adhesion and spreading.

A further assessment was performed using ALP activity to characterize the influence of VA@RGO-nHA on rBMSC osteoblast differentiation with raw RGO sheets as a control (Fig. 9). The ALP enzyme is produced by cells showing a mineralized extracellular matrix, ${ }^{54}$ and it is regarded as an early marker of osteoblast differentiation. ${ }^{55}$ The ALP activity of the rBMSCs was examined on days 0,7 , and 14 , and the results are shown in Fig. 8. It is clear that rBMSCs on RGO, RGO-nHA, and $1 \%$ VA@RGO-nHA experienced an enhancement in the osteogenic differentiation in the culture medium over time. On day 7 , higher levels of ALP activity were detected in 1\% VA@RGO-nHA and RGO-nHA compared to RGO, and this difference was statistically significant on day $14(p<0.05)$. Specifically, there was no evident superiority of RGO-nHA over 1\% VA@RGO-nHA at day 14 ( $p>$ 0.05). The abovementioned result suggested that, compared to the raw RGO sheets, rBMSCs incubated on the RGO-nHA and 1\% VA@RGO-nHA were more osteogenic owing to the nHA incorporation. The similar levels of RGO-nHA and 1\% VA@RGO-nHA at day 14 also indicated that low concentrations of VA did not clearly suppress osteogenic differentiation. The preponderance in cell viability for low concentration VA-loaded RGO-nHA can be
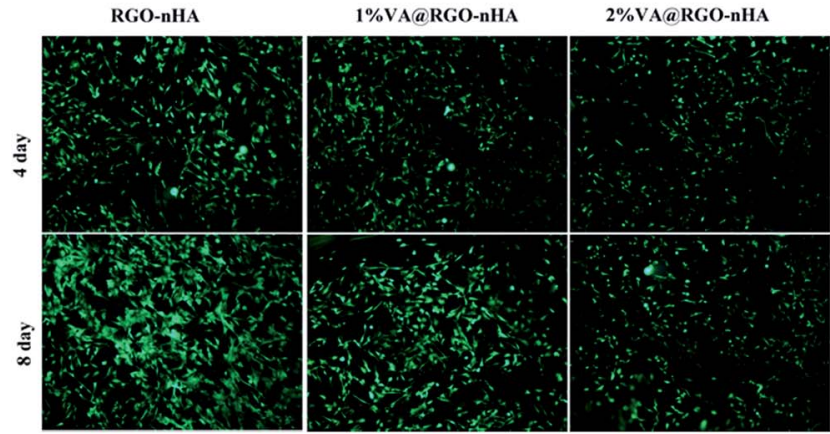

4\%VA@RGO-nHA

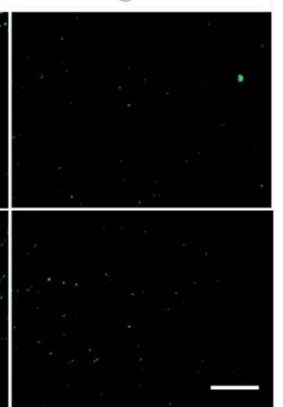

Fig. 6 Fluorescence photographs of rBMSCs incubated with RGO-nHA and VA@RGO-nHA (1\%, 2\%, and $4 \%$ VA) after 4 and 8 days, stained with calcein AM, and visualized under CSLM. Scale bar $=100 \mu \mathrm{m}$. 

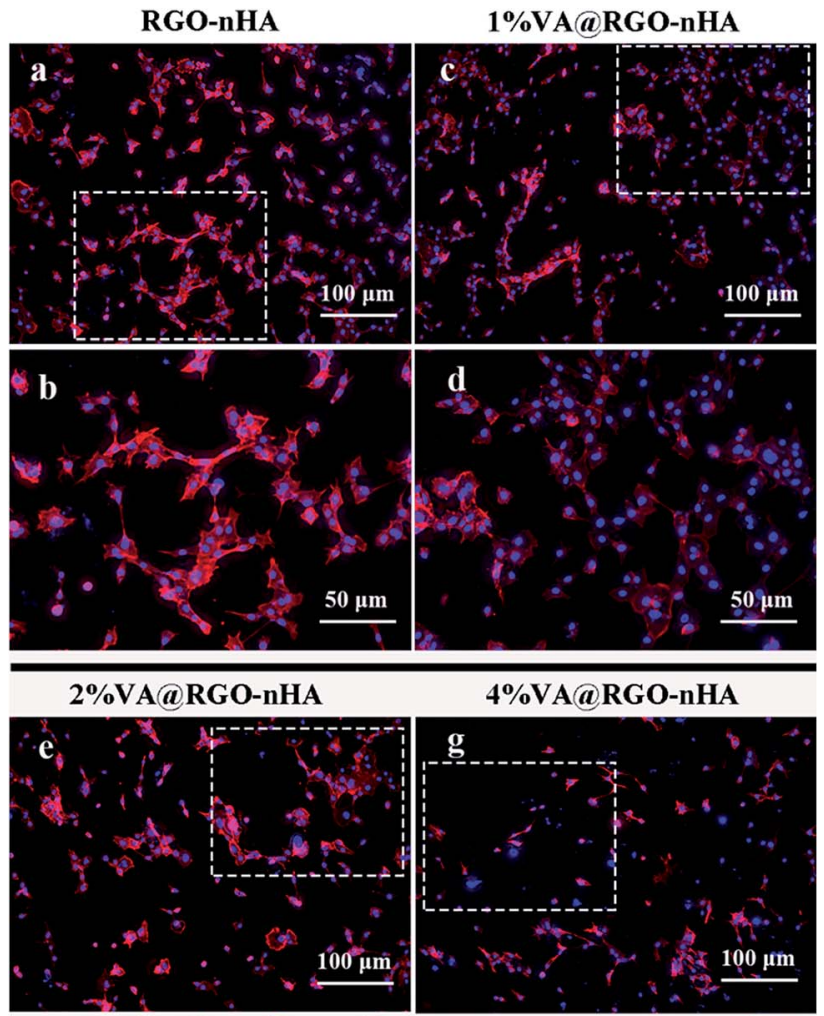

4\%VA@RGO-nHA
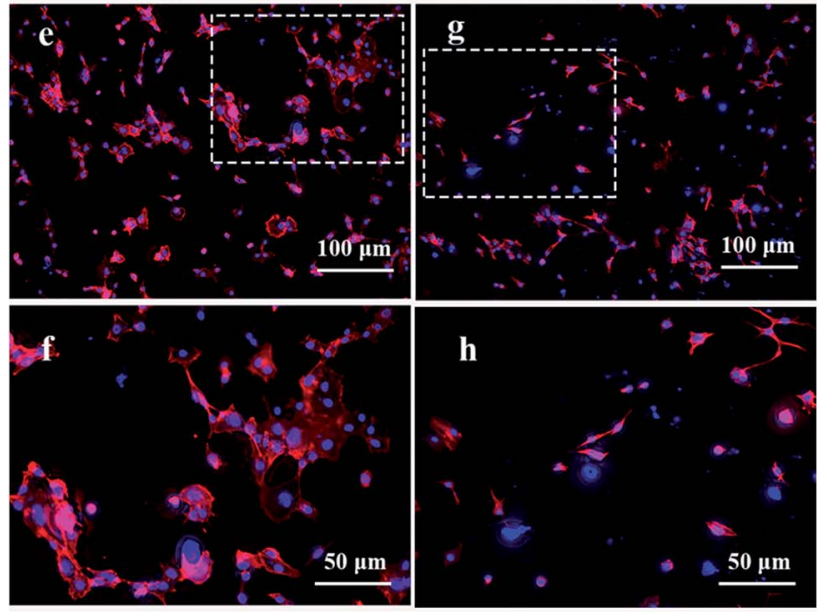

Fig. 7 Fluorescence images of cells cultured for 4 days on $\mathrm{RGO}-\mathrm{nHA}$, $1 \%$ VA@RGO-nHA, 2\% VA@RGO-nHA, and 4\% RGO-nHA scaffolds stained with phalloidin/DAPI and visualized by CLSM. (a, c, e, g) Images under 100x magnification. (b, d, f, h) Images under 200x magnification

explained by the lower cytotoxicity with less VA; more importantly, the sustained release of antibiotics reduces the detrimental effect on osteogenic differentiation..$^{56,57}$

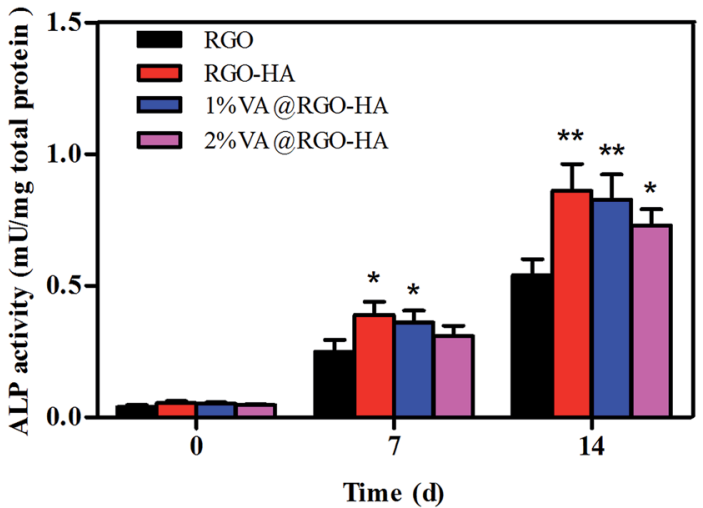

Fig. 9 In vitro ALP activity of rBMSCs cultured on $\mathrm{RGO}-\mathrm{nHA}, 1 \%, 2 \%$, and $4 \%$ VA@RGO-nHA scaffolds for 0,7 , and 14 days. Statistical significance is indicated and compared to the RGO group ( $n=5, * p<$ $0.05 ; * *<0.01)$

\subsection{Antibacterial activity}

We further evaluated the antibacterial activity of the VA@RGO-nHA composite scaffold by comparing different VA concentrations (Fig. 10). The analysis in a liquid medium coculture demonstrated that the antibacterial bioactivity was concentration dependent (Fig. 10a). A higher VA concentration clearly exhibits better bacterial inhibition. The curves indicated that the 4\% VA@RGO-nHA-treated S. aureus barely grew over time, and a satisfactory inhibition performance was also achieved by the 1\% VA@RGO-nHA and $2 \%$ VA@RGO-nHA-treated groups; the bacterial growth was significantly inhibited compared to the control. For the analysis of the solid medium, the photographs of the inhibition zone formed after 1 and 7 days of incubation and the quantitative data are presented (Fig. 10b and c). An obvious inhibition zone formed around all of the scaffolds and remained significant until 7 days (Fig. 10b), suggesting a long-term antibacterial effect owing to the sustained release of VA. Interestingly, although much smaller than that of the VA-loaded groups, the inhibition ring around the RGO-nHA scaffold after 1 day of incubation was apparent, and there were fewer bacteria colony forming units adjacent to the scaffold at 7 days compared to the untreated bacterial growth

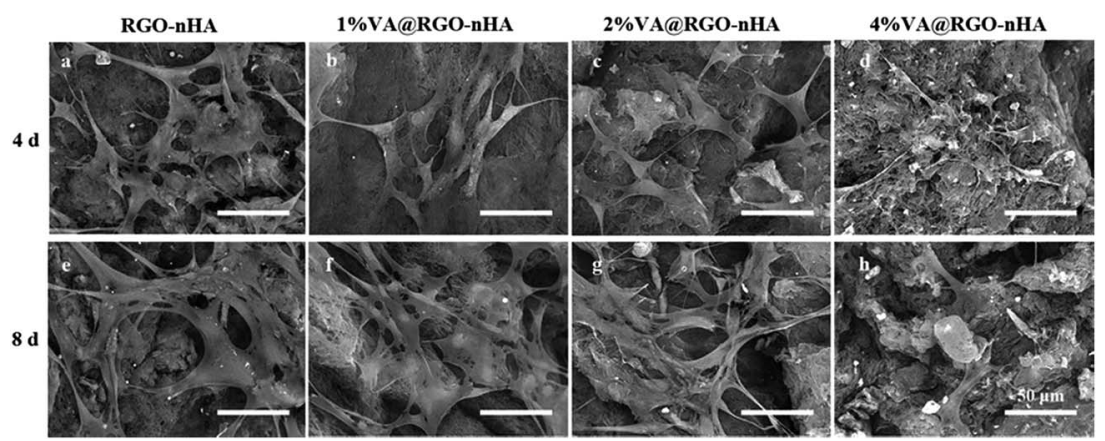

Fig. 8 SEM micrographs of rBMSCs grown onto RGO-nHA, 1\% VA@RGO-nHA, 2\% VA@RGO-nHA, and 4\% VA@RGO-nHA after 4 (a-d) and 8 day $(\mathrm{e}-\mathrm{h})$ cultures. Scale bar $=50 \mu \mathrm{m}$. 
on agar. Previous reports have shown that graphene materials possess inherent antibacterial properties, ${ }^{\mathbf{4 3 , 5 8 - 6 0}}$ which can contribute to this phenomenon.

The antibacterial activity of the scaffolds after 2 days was further studied. As depicted in Fig. 10d, all of the scaffolds showed visible bacterial inhibition rings after 2 days of immersion in the PBS solution, and the specific diameters of the inhibition zones are shown in Fig. 10e. After 2 days, 1\% VA@RGO-nHA presented a larger inhibition zone compared to 4\% VA@RGO-nHA $(p<0.05)$. This likely arose because of the better sustained release of VA from 1\% VA@RGO-nHA and 2\% VA@RGO-nHA, resulting in a higher relative ratio of antibiotics reserved for longer antibacterial bioactivity. The antibacterial results, including liquid and agar medium analyses, clearly suggest that 1\% and 2\% VA@RGO-nHA have longer antibacterial activities, and the inherent antimicrobial capacity of the graphene material adds to its feasibility for biomedical applications.

\subsection{Repair of infected bone defects on the rabbit radius}

After the evaluation of the physicochemical properties, in vitro biocompatibility, and antibacterial capacity, the scaffolds were further assessed in vivo for their efficacy in the treatment of infected bone defects in a rabbit model. Two weeks after modelling surgery, the animal models were verified by infection markers, such as elevated white blood cell count (WBC), Creactive protein (CRP), (Fig. $\mathrm{S} 1 \dagger)$, pus discharge from the wound (Fig. S2 $\dagger$ ), and $S$. aureus bacterial colony formation on the bone marrow sample on agar plates (Fig. S3†). X-ray photographs showed characteristic thickened periosteal reaction and sequestrum in the infected bone (Fig. $\mathrm{S} 4 \dagger$ ), and histological examination further indicated inflammatory cell infiltration and lack of intact morphology on the damaged trabecula bone (Fig. S5†).

The rabbits were first treated with debridement and then randomly divided into three groups for implantation. The scaffold loaded with 1\% VA was used in this experiment because of its ideal biocompatibility, osteoinductivity, and satisfactory

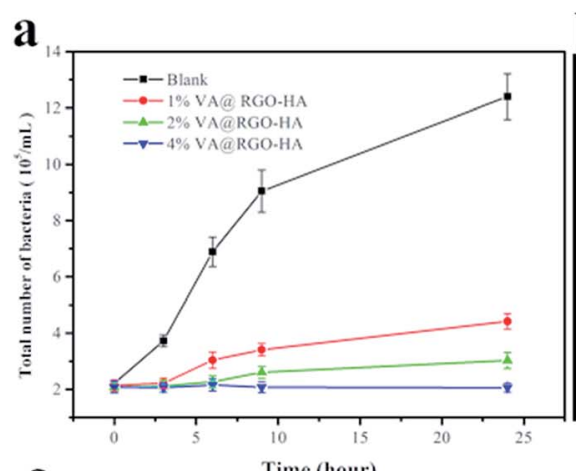

b
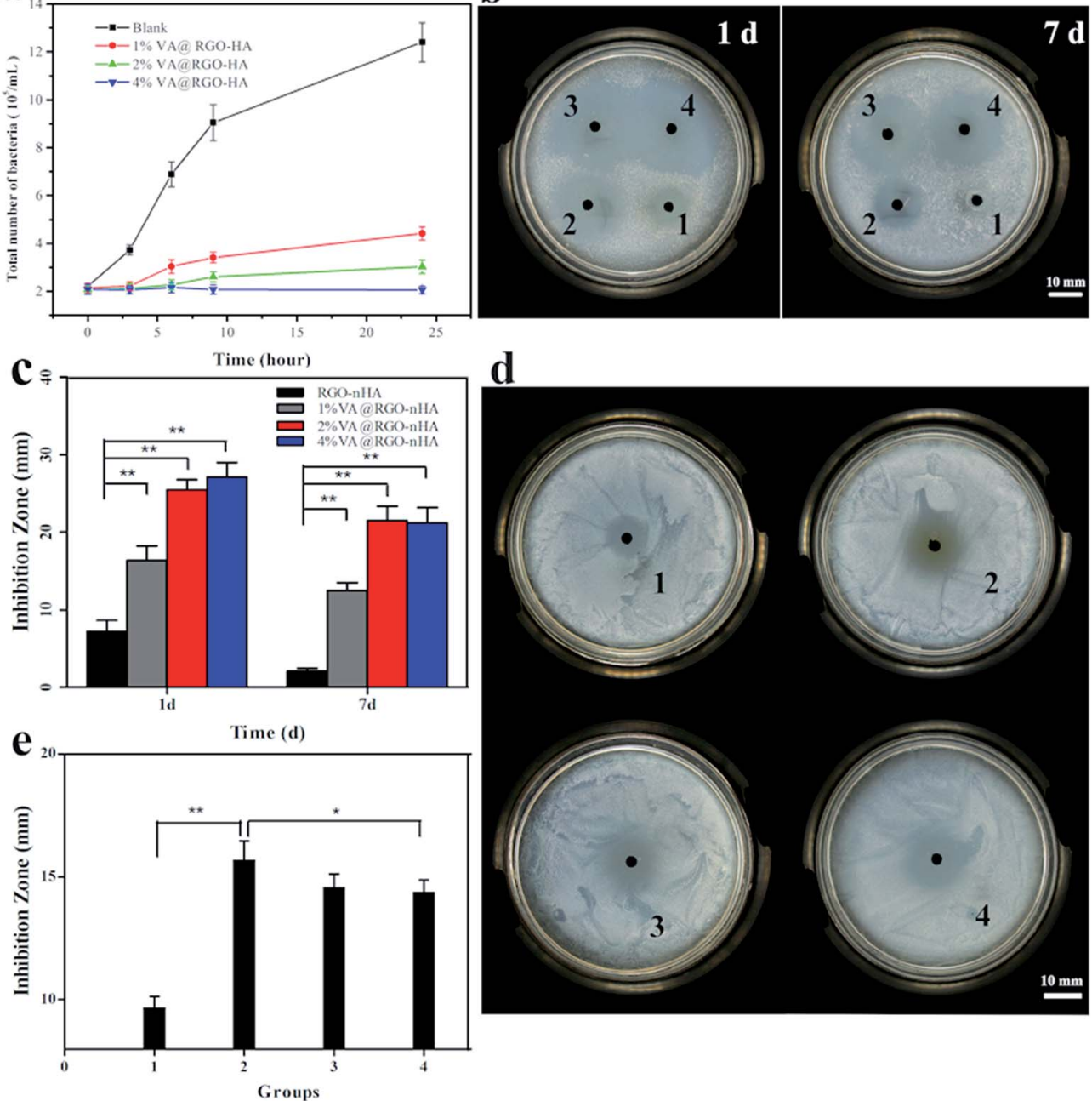

Fig. 10 Antibacterial evaluation in liquid and solid media. (a) Bacterial growth in a liquid medium co-cultured with 1\%, 2\%, and 4\% VA@RGO-nHA scaffolds. The blank was set as the control. (b, c) Bacterial inhibition of the scaffolds without immersion in PBS on a solid medium at 1 and 7 days. (d, e) Growth inhibition of bacteria on a solid medium at an incubation time of $24 \mathrm{~h}$ after a 2 day release. Spots 1-4 represent RGO-nHA, 1\% VA@RGO-nHA, 2\% VA@RGO-nHA, and 4\% VA@RGO-nHA, respectively. $(n=5, * p<0.05 ; * *<0.01)$. 

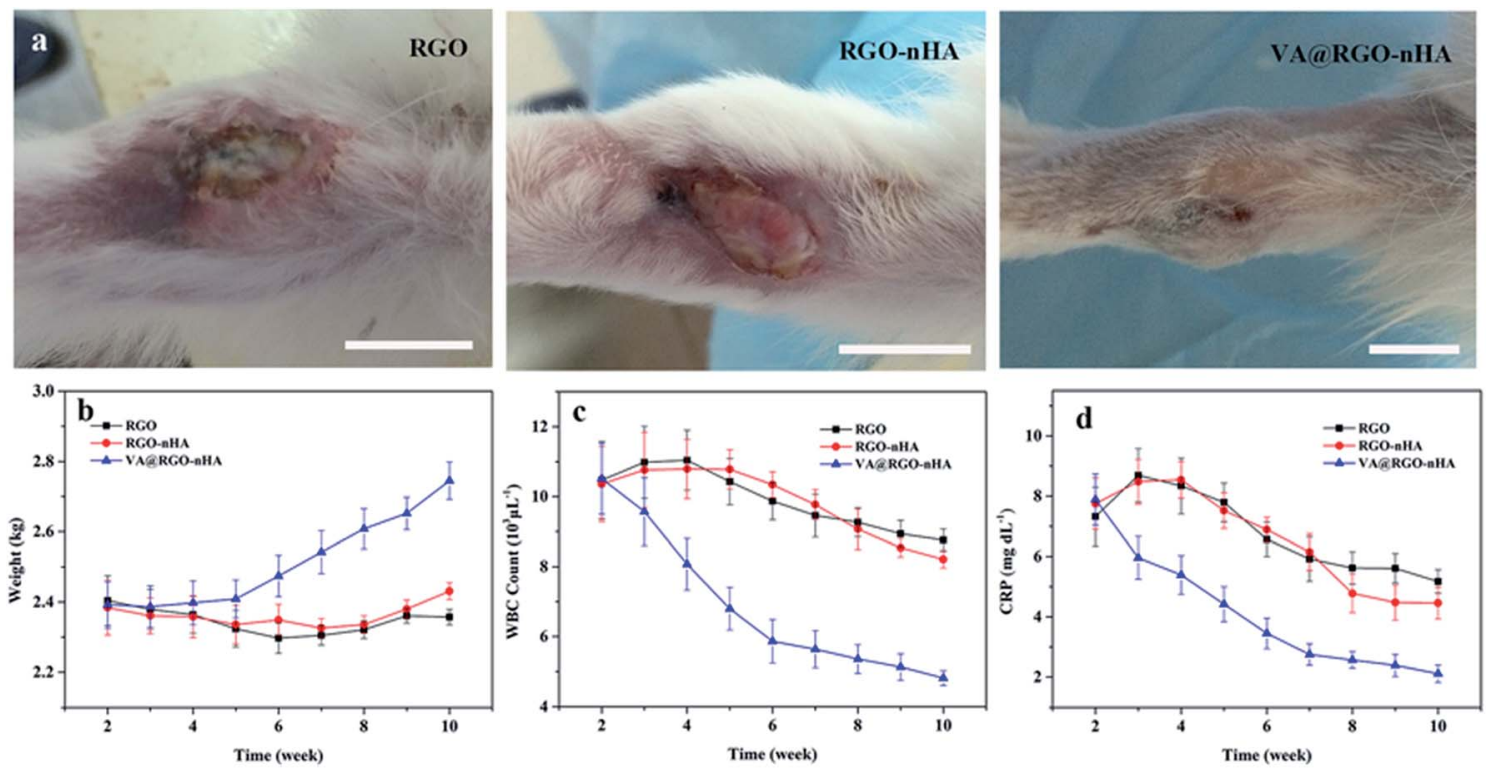

Fig. 11 (a) Digital photographs of the rabbit forearms treated with scaffolds after 4 weeks. Scale bar $=10 \mathrm{~mm}$. (b) Changes in body weight. (c) Changes in WBC count. (d) Changes in CRP levels of rabbits implanted with different scaffolds.

antibacterial activity; RGO and RGO-nHA scaffolds were used for comparison.

During the post-implantation investigation, the wound observation revealed better healing in the VA@RGO-nHA group after intervention without obvious pus discharge (Fig. 11a). The weekly measured weights of the rabbits after implantation are shown in Fig. 11b. During the first week after intervention, decreased animal weights were detected in all groups owing to
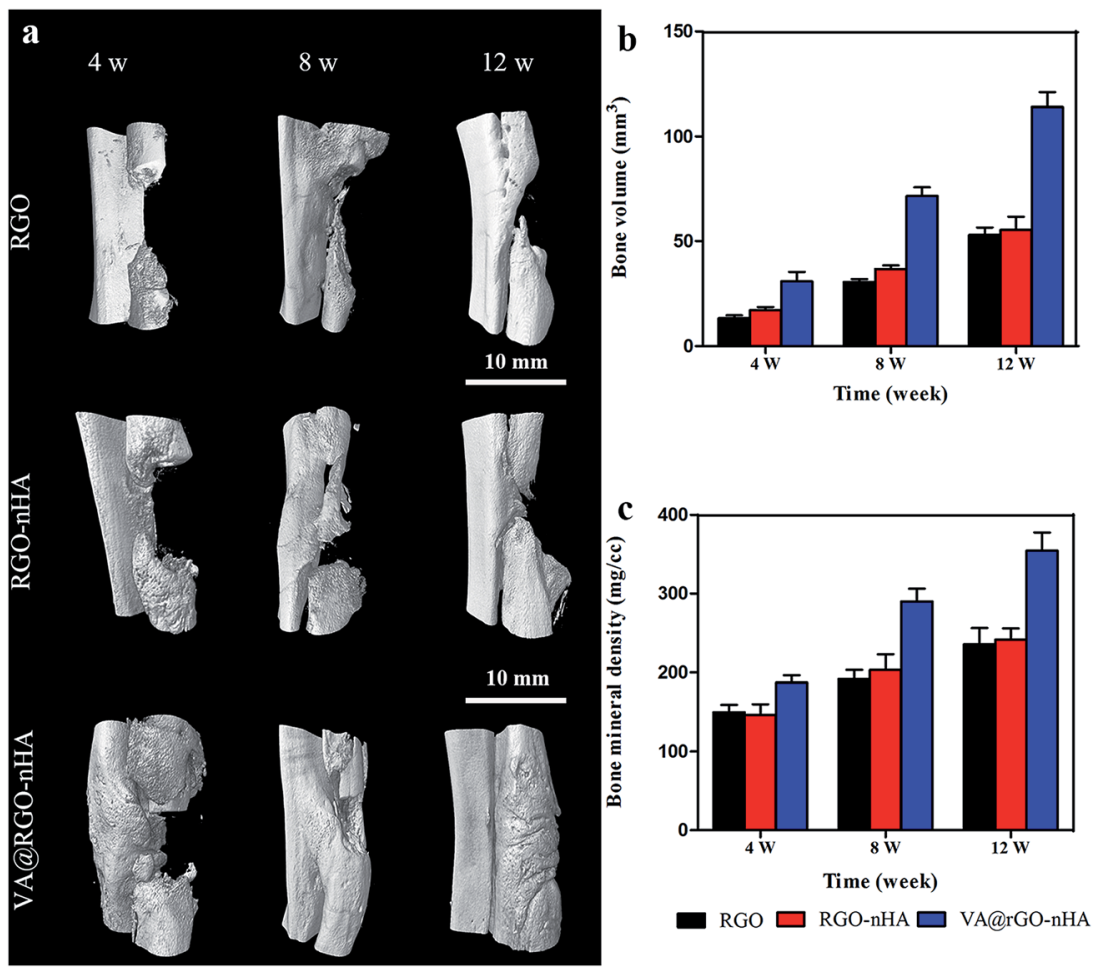

Fig. 12 Imaging assessment of infected bone defects implanted with different scaffolds. (a) Micro-CT evaluation of the rabbit infected bone defects implanted with RGO, RGO-nHA and VAaRGO-nHA at 4, 8, and 12 weeks, respectively. Scale bar $=10 \mathrm{~mm}$. (b) Quantitative analysis based on the extracted data of regenerated bone volume from the Micro-CT images at 4, 8, and 12 weeks, $n=5 / g r o u p$. (c) Quantitative analysis based on the extracted data of bone mineral density at 4,8 , and 12 weeks, $n=5 /$ group. 
post-operational immobilization. A recovery in weight was observed in the VA@RGO-nHA group in the following weeks. The rabbits in the RGO and the RGO-nHA groups suffered from prolonged incommodity in the affected limbs, and they weighed significantly less $(p<0.05)$. Blood samples were also retrieved at predetermined time intervals with ethylenediaminetetraacetic acid (EDTA) tubes for analysis of the WBC and CRP. Variation in these infection parameters demonstrated a distinguishing difference between the groups (Fig. 11c and d). One week after implantation, the RGO and the RGO-nHA groups both had high WBC and CRP levels owing to foreign body reactions and a deficiency of antibiotics. Following an investigation over time, the WBC and CRP indicators for the non-drug loading groups remained higher than the VA@RGO-nHA group $(p<0.05)$, and the levels in the VA@RGO-nHA group declined over time and returned to a normal range 5 weeks after treatment. The difference between the groups is because while graphene materials possess some inherent antibacterial activity, ${ }^{\mathbf{4 2 , 4 3}}$ it was not adequate for satisfactory infection control without antimicrobial agents in the present study. The comparison between the groups revealed the effective treatment of the VA@RGOnHA group infections.

An imaging evaluation was further conducted with a microCT on bone specimens obtained at 4, 8, and 12 weeks after implantation (Fig. 12). The group implanted with the RGO exhibited the slowest bone bridging and regeneration rate with no obvious neo-bone formation until 8 weeks, which was mainly attributed to the bacterial colonization and the non-porous structure of the RGO sheets. The RGO-nHA group had slightly better bone regeneration but was far from satisfactory, which can be explained by the "race to the surface theory" 61 that bone regeneration requires a high level of sterility and bacteria can seriously hinder the bone regeneration process despite the presence of osteoinductive components. ${ }^{62}$ Although the a

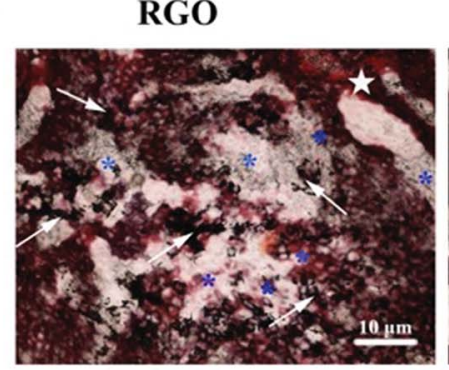

8 w

$12 \mathrm{w}$

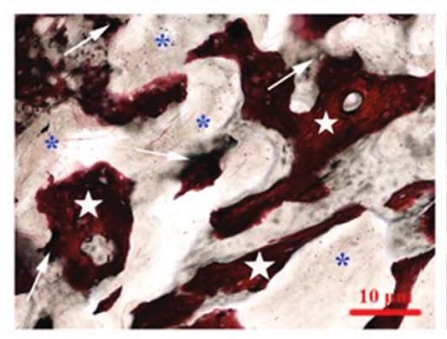

b

$8 \mathbf{w}$
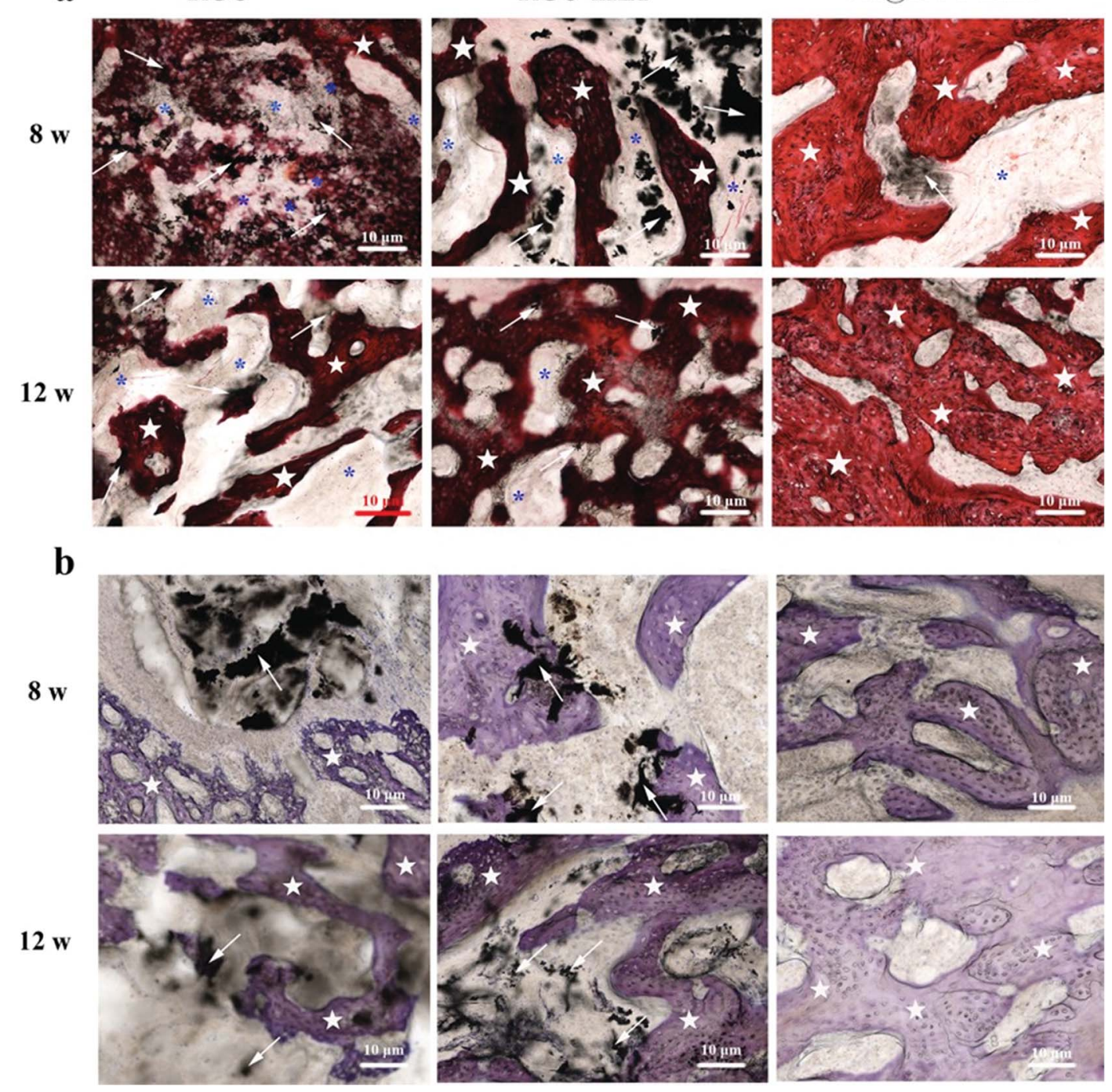

Fig. 13 Histological photomicrographs of the undecalcified sections of the retrieved bone specimens stained with (a) H\&E and (b) T-blue at 8 and 12 weeks after implantation of different scaffolds. Stars indicate new regenerated bone, arrows indicate residual materials, and asterisks indicate inflammatory cells. 
composite incorporated nHA can theoretically improve the osteogenesis performance, ${ }^{47,63}$ its efficacy was suppressed by bacterial propagation. In contrast, groups implanted with VA@RGO-nHA showed satisfactory bone binding in the defect sites because of their ability to provide a sustained release of VA for the effective treatment of the infection and enhanced bone repair due to nHA incorporation. In particular, there were higher density areas observed at the defect site in the VA@RGOnHA group at 8 weeks and up to 12 weeks compared to the other groups. The micro-CT images showed solid bone bridging within the voids. Unsatisfactory bone formation was observed in the RGO and RGO-nHA groups with unrepaired bone defects until 12 weeks. The regenerated bone volume (BV) and the bone mineral density (BMD) were further extracted for quantitative analysis (Fig. 12b and c). The highest BV and BMD values were observed in the VA@RGO-nHA scaffolds at 4, 8, and 12 weeks, while the RGO group demonstrated the lowest regenerated BV and BMD values without nHA to improve the osteoconductivity or VA for infection control.

Further verification was obtained via a histological analysis with H\&E and Toluidine-blue (T-blue) staining; the sagittal undecalcified sections at 8 and 12 weeks post-implantation are shown in Fig. 13. H\&E-stained sections showed the presence of inflammatory cell infiltration in the vicinity of the affected bone trabeculae at 8 and 12 weeks in the RGO and RGO-nHA groups, and there were also obvious residual materials (Fig. 13a). For the VA@RGO-nHA group, the tissue inflammation was dramatically reduced, and more regenerated bone trabeculae were observed with an intact morphology. The T-blue staining offered a more specific visualization of the new bone formation. The stained sections showed more regenerated osseous tissues in the VA@RGO-nHA scaffold groups at 8 and 12 weeks postimplantation, and there were also significantly less residual materials in the vicinity of the new bones compared to the RGO and RGO-nHA groups (Fig. 13b). These histological results confirmed that the VA@RGO-nHA scaffold, owing to its welldesigned porous structure and biocompatible characteristics, facilitated cell ingrowth and adhesion, and the addition of nHA presented better osteoconductivity and osteoinductivity. Moreover, the loading of VA onto the scaffold presented better antibacterial activity and, as a whole, effectively treated the infected bone defect.

\section{Conclusion}

In conclusion, the VA-loaded RGO-nHA scaffold was successfully prepared via a self-assembly method. The highly interconnected 3D porous architecture of the scaffold was characterized. The drug delivery system was established using the unique physicochemical properties of graphene, such as a large surface area and $\pi$-electron-rich structure, which demonstrated a sustained release profile due to the $\pi-\pi$ stacking interaction between VA and RGO. More importantly, a rational loading ratio of VA in this strut was highlighted with the extended drug release duration, achieving an efficient and prolonged antibacterial activity together with the inherent antimicrobial ability of RGO. Moreover, VA at this loading concentration did not significantly compromise the enhanced cell viability contributed by the combination of nHA and RGO as well as the 3D porous structure of this system. Moreover, the in vivo evaluation in an infected bone defect animal model indicated that the VA@RGO-nHA scaffold possessed an on-demand potential for the treatment of infected bone defects. In any case, our study expanded new strategies for fabricating graphene into a multifunctional drug delivery system and demonstrated that graphene-based composites with antibiotics can provide new approaches to treating intractable orthopedic diseases.

\section{Conflict of interest}

The authors declare no competing financial interest.

\section{Acknowledgements}

This study was financially supported by the National Natural Science Foundation of China (31271031, 312111282), Major Sino-Korea joint research programme of the National Natural Science Foundation (8141101156), and the fundamental research project of the Science and Technology Commission of Shanghai Municipality (15411950600).

\section{References}

1 T. A. DeCoster, R. J. Gehlert, E. A. Mikola and M. A. PirelaCruz, J. Am. Acad. Orthop. Surg., 2004, 12, 28-38.

2 P. A. Ostermann, S. L. Henry and D. Seligson, Clin. Orthop. Relat. Res., 1993, 102-111.

3 M. J. Bosse, E. J. MacKenzie, J. F. Kellam, A. R. Burgess, L. X. Webb, M. F. Swiontkowski, R. W. Sanders, A. L. Jones, M. P. McAndrew, B. M. Patterson, M. L. McCarthy, T. G. Travison and R. C. Castillo, N. Engl. J. Med., 2002, 347, 1924-1931.

4 A. M. Abdel-Aal, Orthopedics, 2006, 29, 70-74.

5 I. Keren, N. Kaldalu, A. Spoering, Y. Wang and K. Lewis, FEMS Microbiol. Lett., 2004, 230, 13-18.

6 M. P. Ginebra, T. Traykova and J. A. Planell, J. Controlled Release, 2006, 113, 102-110.

7 M. Zilberman and J. J. Elsner, J. Controlled Release, 2008, 130, 202-215.

8 P. Zhou, Y. Xia, X. Cheng, P. Wang, Y. Xie and S. Xu, Biomaterials, 2014, 35, 10033-10045.

9 R. L. Stewart, J. T. Cox, D. Volgas, J. Stannard, L. Duffy, K. B. Waites and T. M. Chu, J. Orthop. Traumatol., 2010, 24, 587-591.

10 A. C. Matos, C. F. Marques, R. V. Pinto, I. A. Ribeiro, L. M. Goncalves, M. A. Vaz, J. M. Ferreira, A. J. Almeida and A. F. Bettencourt, Int. J. Pharm., 2015, 490, 200-208.

11 B. D. Brooks, K. D. Sinclair, D. W. Grainger and A. E. Brooks, PLoS One, 2015, 10, e0118696.

12 C. P. Duncan and B. A. Masri, Instr. Course. Lect., 1995, 44, 305-313.

13 C. L. Nelson, R. P. Evans, J. D. Blaha, J. Calhoun, S. L. Henry and M. J. Patzakis, Clin. Orthop. Relat. Res., 1993, 96-101.

14 A. D. Hanssen and M. J. Spangehl, Clin. Orthop. Relat. Res., 2004, 79-85. 
15 J. Min, K. Y. Choi, E. C. Dreaden, R. F. Padera, R. D. Braatz, M. Spector and P. T. Hammond, ACS Nano, 2016, 10, 4441-4450.

16 B. S. Lee, C. C. Lee, Y. P. Wang, H. J. Chen, C. H. Lai, W. L. Hsieh and Y. W. Chen, Int. J. Nanomed., 2016, 11, 285-297.

17 K. Feng, H. Sun, M. A. Bradley, E. J. Dupler, W. V. Giannobile and P. X. Ma, J. Controlled Release, 2010, 146, 363-369.

18 W. Liu, J. Wei, Y. Wei and Y. Chen, J. Biomed. Nanotechnol., 2015, 11, 428-435.

19 S. Bhattacharyya, A. Agrawal, C. Knabe and P. Ducheyne, Biomaterials, 2014, 35, 509-517.

20 M. P. Ferraz, A. Y. Mateus, J. C. Sousa and F. J. Monteiro, J. Biomed. Mater. Res., Part A, 2007, 81, 994-1004.

21 B. Kundu, C. Soundrapandian, S. K. Nandi, P. Mukherjee, N. Dandapat, S. Roy, B. K. Datta, T. K. Mandal, D. Basu and R. N. Bhattacharya, Pharm. Res., 2010, 27, 1659-1676.

22 A. El-Ghannam, K. Jahed and M. Govindaswami, J. Biomed. Mater. Res., Part A, 2010, 94, 308-316.

23 X. Shi, Y. Wang, L. Ren, N. Zhao, Y. Gong and D. A. Wang, Acta Biomater., 2009, 5, 1697-1707.

24 S. L. Percival, K. E. Hill, S. Malic, D. W. Thomas and D. W. Williams, Wound Repair Regen., 2011, 19, 1-9.

25 W. C. Lee, C. H. Lim, H. Shi, L. A. Tang, Y. Wang, C. T. Lim and K. P. Loh, ACS Nano, 2011, 5, 7334-7341.

26 T. R. Nayak, H. Andersen, V. S. Makam, C. Khaw, S. Bae, X. Xu, P. L. Ee, J. H. Ahn, B. H. Hong, G. Pastorin and B. Ozyilmaz, ACS Nano, 2011, 5, 4670-4678.

27 H. Liu, J. Cheng, F. Chen, F. Hou, D. Bai, P. Xi and Z. Zeng, ACS Appl. Mater. Interfaces, 2014, 6, 3132-3140.

28 Y. Luo, H. Shen, Y. Fang, Y. Cao, J. Huang, M. Zhang, J. Dai, X. Shi and Z. Zhang, ACS Appl. Mater. Interfaces, 2015, 7, 6331-6339.

29 H. Liu, J. Cheng, F. Chen, D. Bai, C. Shao, J. Wang, P. Xi and Z. Zeng, Nanoscale, 2014, 6, 5315-5322.

30 X. Xie, K. Hu, D. Fang, L. Shang, S. D. Tran and M. Cerruti, Nanoscale, 2015, 7, 7992-8002.

31 X. Sun, Z. Liu, K. Welsher, J. T. Robinson, A. Goodwin, S. Zaric and H. Dai, Nano Res., 2008, 1, 203-212.

32 D. Depan, J. Shah and R. D. K. Misra, Mater. Sci. Eng., C, 2011, 31, 1305-1312.

33 B. Ardeshirzadeh, N. A. Anaraki, M. Irani, L. R. Rad and S. Shamshiri, Mater. Sci. Eng., C, 2015, 48, 384-390.

34 L. Zhang, J. Xia, Q. Zhao, L. Liu and Z. Zhang, Small, 2010, 6, 537-544.

35 S. Silverman, J. Moses and K. B. Sharpless, Chemistry, 2016, DOI: $10.1002 /$ chem.201604765.

36 S. W. Ueng, S. S. Lin, I. C. Wang, C. Y. Yang, R. C. Cheng, S. J. Liu, E. C. Chan, C. F. Lai, L. J. Yuan and S. C. Chan, J. Orthop. Surg. Res., 2016, 11, 52.

37 T. Hui, X. Yongqing, Z. Tiane, L. Gang, Y. Yonggang, J. Muyao, L. Jun and D. Jing, Arch. Orthop. Trauma Surg., 2009, 129, 1301-1308.

38 J. A. Inzana, E. M. Schwarz, S. L. Kates and H. A. Awad, Biomaterials, 2016, 81, 58-71.

39 J. M. Lestner, L. F. Hill, P. T. Heath and M. Sharland, Curr. Opin. Infect. Dis., 2016, 29, 237-247.
40 T. Ringenberg, C. Robinson, R. Meyers, L. Degnan, P. Shah, A. Siu and M. Sturgill, J. Pediatr. Infect. Dis., 2015, 34, 742747.

41 F. Khalilian, S. A. Hanzaki and M. Yousefi, J. Sep. Sci., 2015, 38, 975-981.

42 O. Akhavan and E. Ghaderi, ACS Nano, 2010, 4, 5731-5736.

43 S. Liu, T. H. Zeng, M. Hofmann, E. Burcombe, J. Wei, R. Jiang, J. Kong and Y. Chen, ACS Nano, 2011, 5, 6971-6980.

44 S. Kim, S. H. Ku, S. Y. Lim, J. H. Kim and C. B. Park, Adv. Mater., 2011, 23, 2009-2014.

45 G. M. Neelgund, A. Oki and Z. Luo, Mater. Res. Bull., 2013, 48, 175-179.

46 J. H. Lee, Y. C. Shin, O. S. Jin, S. H. Kang, Y. S. Hwang, J. C. Park, S. W. Hong and D. W. Han, Nanoscale, 2015, 7, 11642-11651.

47 M. Nair, D. Nancy, A. G. Krishnan, G. S. Anjusree, S. Vadukumpully and S. V. Nair, Nanotechnology, 2015, 26, 161001.

48 L. Ding, X. Li, H. Sun, J. Su, N. Lin, B. Peault, T. Song, J. Yang, J. Dai and Y. Hu, Biomaterials, 2014, 35, 4888-4900.

49 N. Hild, O. D. Schneider, D. Mohn, N. A. Luechinger, F. M. Koehler, S. Hofmann, J. R. Vetsch, B. W. Thimm, R. Muller and W. J. Stark, Nanoscale, 2011, 3, 401-409.

50 K. Kim, Y. K. Luu, C. Chang, D. Fang, B. S. Hsiao, B. Chu and M. Hadjiargyrou, J. Controlled Release, 2004, 98, 47-56.

51 H. Li, T. Hamza, J. E. Tidwell, N. Clovis and B. Li, Adv. Healthcare Mater., 2013, 2, 1277-1284.

52 U. B. Suryavanshi, T. Ijima, A. Hayashi, Y. Hayashi and M. Tanemura, Chem. Commun., 2011, 47, 10758-10760.

53 M. F. Chung, W. T. Chia, H. Y. Liu, C. W. Hsiao, H. C. Hsiao, C. M. Yang and H. W. Sung, Adv. Healthcare Mater., 2014, 3, 1854-1861.

54 G. S. Stein, J. B. Lian and T. A. Owen, FASEB J., 1990, 4, 31113123.

55 Y. Gotoh, K. Hiraiwa and M. Nagayama, Bone Miner., 1990, 8, 239-250.

56 Z. Zhang, S. Liu, H. Xiong, X. Jing, Z. Xie, X. Chen and Y. Huang, Acta Biomater., 2015, 26, 115-123.

57 G. Li, L. Cao, Z. Zhou, Z. Chen, Y. Huang and Y. Zhao, Colloids Surf., B, 2015, 128, 379-388.

58 S. Gurunathan, J. W. Han, V. Eppakayala and J. H. Kim, Colloids Surf., B, 2013, 105, 58-66.

59 I. E. Mejias Carpio, C. M. Santos, X. Wei and D. F. Rodrigues, Nanoscale, 2012, 4, 4746-4756.

60 H. Sun, N. Gao, K. Dong, J. Ren and X. Qu, ACS Nano, 2014, 8, 6202-6210.

61 A. G. Gristina, P. Naylor and Q. Myrvik, Med. Prog. Technol., 1988, 14, 205-224.

62 S. A. Guelcher, K. V. Brown, B. Li, T. Guda, B. H. Lee and J. C. Wenke, J. Orthop. Traumatol., 2011, 25, 477-482.

63 J. H. Lee, Y. C. Shin, S. M. Lee, O. S. Jin, S. H. Kang, S. W. Hong, C. M. Jeong, J. B. Huh and D. W. Han, Sci. Rep., 2015, 5, 18833. 